Mariona Grané

Universitat de Barcelona (UB)

E-mail:mgrane@ub.edu

(D) https://orcid.org/ 0000-0002-1435-0664

Recebido em: 15/07/2019

Aprovado em: 29/04/2020

\section{Pantallas digitales para infancias diversas}

Mariona Grané

\section{Resumen}

Familias y maestros de todos los países dedican esfuerzos en encontrar recursos digitales (hoy sobre todo juegos y Apps para dispositivos móviles) que ayuden a sus niños y niñas a jugar y a aprender. Para todos los educadores es importante acceder a recursos de calidad, pero todavía lo es más para aquellos que trabajan con niños con diversidad funcional, porque necesitan además recursos muy específicos y el mercado les ofrece materiales diversos, pero no siempre con la calidad adecuada. Diversos estudios sobre las potencialidades de estos materiales digitales en los procesos de aprendizaje durante la infancia de los menores con o sin necesidades educativas especiales, contrastan con los resultados de investigaciones acerca de la calidad real de los recursos existentes. Ello requiere de un necesario avance en la consideración del diseño interactivo de los juegos y Apps para este target. Este artículo muestra los resultados de cuatro diferentes investigaciones sobre la calidad de Apps para niños y niñas menores de 8 años, con desarrollo optimo y con necesidades educativas especiales; y plantea propuestas que orienten una creación de Apps interactivas que permitan a todos jugar y aprender. Porque la finalidad de esta línea de investigación es determinar la calidad en los recursos interactivos orientados a la primera infancia ( $0-8$ años), considerando el contenido, pero también su forma, el diseño de este contenido.

Palabras clave: Apps. Infancia. Diversidad. 


\section{Resumo}

Palavras-chave:

Apps.

Infância.

Diversidade.

\section{Telas digitais para diversas infâncias}

Famílias e professores de todo o mundo dedicam grandes esforços para encontrar recursos digitais (hoje especialmente jogos e aplicativos para smartphones e tablets) para ajudar meninos e meninas a brincar e a aprender. Para todos os educadores, é importante o acesso a recursos de qualidade, mas é ainda mais importante para quem trabalha com crianças com diversidade funcional, pois precisam de recursos muito específicos. $\mathrm{O}$ mercado oferece diferentes materiais para essas crianças, mas nem sempre com a qualidade adequada. Existem vários estudos sobre o potencial desses materiais digitais nos processos de aprendizagem durante a infância, mas esses estudos contrastam com os resultados de pesquisas sobre a qualidade real de aplicativos e jogos de vídeo para crianças. É necessário avançar na avaliação da qualidade do conteúdo e no design interativo dos jogos e aplicativos para todas as crianças. Este artigo mostra os resultados de diferentes análises sobre a qualidade do Apps para crianças menores de 8 anos, com ótimo desenvolvimento e com necessidades educacionais especiais, utilizando diferentes metodologias; e apresenta propostas para orientar a seleção e criação de aplicativos interativos que permitem que todas as crianças brinquem e aprendam. O objetivo desta linha de pesquisa é determinar a qualidade dos recursos interativos voltados para a primeira infância (0-8 anos), considerando o conteúdo, mas também a forma, o design deste conteúdo.

\begin{abstract}
Digital screens for diverse childhoods

Families and teachers from all around the world dedicate big efforts to find digital resources to help their children to play and learn, today especially games and Apps for smartphones and tablets. For all educators, it is important the access of quality resources, but it is even more important for those who work with children with functional diversity because they also need very specific resources and the market offers them different materials but not always with the adequate quality needed by these children. There are several studies on the potential of these digital materials in learning processes during childhood, but these studies contrast with the results of research about the real quality of Apps and video games for children. It is necessary to advance in the evaluation of the content quality and the interactive design of the games and Apps for all kids. This article shows the results of four different analyses on the quality of Apps for children under 8, with optimal development and with special educational needs (SEN), using different methodologies; and presents proposals to guide the selection and creation of interactive Apps that allow all children to play and learn. Because the purpose of this line of research is to determine the quality of interactive resources aimed at early childhood considering the content but also its form, the design of this content.

Keywords:

Apps.

Childhood.

Diversity.
\end{abstract}




\section{Introducción y estado de la cuestión}

Todos los niños y niñas quieren jugar, aprender y crecer. Y todos deberían tener la oportunidad de hacerlo desde sus habilidades y desde sus potencialidades. Por ello, familias y educadores de todo el mundo dedican mucho tiempo a buscar y seleccionar recursos de calidad que ayuden a los menores en su desarrollo cognitivo, psicomotor y emocional. Esta selección se da en diferentes ámbitos (recursos didácticos, juegos y juguetes, libros, música, ...) pero hoy especialmente con recursos digitales tales como Apps y juegos para dispositivos móviles (tablets y smartphones).

Nuestros niños y niñas se han apropiado de estas herramientas y las han integrado en su vida como ninguna otra generación había hecho antes con ninguna otra tecnología (READ Y MARKOPOULOS, 2013). Ellos son los usuarios más apasionados de móviles, Apps y videojuegos, pero, sin embargo, también son los menos expertos y críticos con los recursos que utilizan.

Los datos indican que el uso de dispositivos móviles, casi siempre conectados a Internet, ha estado creciendo imparablemente en familias de todo el mundo desde el 2010 con la irrupción de las primeras tablets (iPad) en el mercado. Ya en 2014, diferentes estudios en América y Europa mostraban una tendencia creciente del uso de dispositivos móviles en los hogares y de la mano de los menores. Los datos del informe EU Kids online (2014), referentes a la adopción de las tecnologías en Europa por parte de niños y jóvenes, mostraban conexiones a Internet a diario, desde cualquier lugar, con diversos dispositivos (pero especialmente móviles) y cada vez a una edad más temprana (MASCHERONI Y CUMAN, 2014; EU KIDS ONLINE, 2014). Además, el mismo año, el estudio del Reynolds Journalism Institute (FIDLER, 2014) mostró que los hogares con niños tenían más dispositivos móviles que los hogares sin menores. Los resultados coinciden con aquellos encontrado por Vincent (2015), que revelaban como el número de niños y niñas que se conectan habitualmente a Internet desde dispositivos móviles se había doblado en la mayoría de los países en dos años (entre 2013 y 2015).

Y año tras año, esta tendencia se ha mantenido. El Mobile Kids Report del Nielsen Group (2017), que encuestó a 4.646 familias con niños de entre 6 y 12 años, mostró que casi la mitad (45\%) obtiene su primer móvil entre los 10 y los 12 años, pero también hay menores de 10 años con móvil propio. El 72\% de estos niños tiene todos los servicios incluidos (voz, mensajes y datos). A pesar de ser los responsables de ello, un $71 \%$ de los padres se muestra preocupado y un $68 \%$ ve alarmante el hecho de no controlar los contenidos que sus hijos utilizan desde sus móviles. El estudio también mostró que todos los menores tenían acceso a Internet, jugaban con aplicaciones en sus dispositivos y un 59\% de los niños instalaban ellos mismos las Apps en sus smartphones sin necesidad de control paterno.

El último estudio de Global Kids online se está realizando desde 2017 en diferentes países europeos e incluyendo países que no pertenecen a la Unión Europea como Brasil, Argentina, Chile, Serbia, Sud 
África, Filipinas, Bulgaria y Montenegro. En Brasil la encuesta ICT Kids Online Brazil (2017) estima que aproximadamente ocho de cada diez niños (85\%) de 9 a 17 años son usuarios de Internet, lo que corresponde a 24.7 millones de usuarios en todo el país. Este dato es relevante porque en 2016 esta proporción fue del 82\% (aproximadamente 23 millones). La encuesta también muestra un aumento en el uso de teléfonos móviles entre los niños para acceder a Internet en los últimos años. En 2012, el 21\% de los niños se conectaron a través de un teléfono móvil, mientras que en 2017-18 este porcentaje es del 93\%, lo que equivale a 23 millones de niños brasileños que se conectan a la red a partir del móvil. Y al mismo tiempo, hay un $37 \%$ de disminución en el uso de otros dispositivos, como ordenadores y tablets, para acceder a Internet, del 90\% de los niños en 2013 al 53\% en 2018. La encuesta también estima que el 44\% de los usuarios de Internet infantiles se conectan exclusivamente a través de un teléfono móvil, lo que representa a 11 millones de personas jóvenes del país. Los teléfonos móviles son el principal dispositivo de acceso a internet para niños en áreas rurales (57\%), en el norte del país (59\%) y de bajos niveles socioeconómicos $(67 \%)$.

En sí mismos estos datos no constituyen un problema. Como todos los medios, los digitales son, potencialmente, una oportunidad. Son herramientas que pueden apoyar el aprendizaje y el desarrollo infantil, como indica la literatura actual. En comparación con los medios de comunicación tradicionales que mantienen a los niños pasivos consumiendo contenidos audiovisuales, diferentes investigaciones han demostrado que el uso de tecnologías interactivas (desde ordenadores a pantallas táctiles) por parte de niños pequeños, promueve el aprendizaje de una forma significativa (LAURICELLA ET AL., 2010; KIRKORIAN \& PEMPEK, 2013; FLEWITT, MESSER \& KUCIRKOVA, 2014). Los niños que utilizan juegos interactivos tienen una mayor habilidad para la solución de problemas, en comparación con los que no utilizan la tecnología (CLEMENTS \& SAMARA, 2003; VERNADAKIS ET AL., 2005). Además, Kirkorian y Pempek (2013) observaron que los niños de 2 a 3 años progresan más rápidamente, se equivocan menos y aprenden más ante pantallas interactivas en comparación con aquellas que los mantienen pasivos (como la televisión).

Ante ello, la búsqueda constante de padres y educadores, de estos recursos interactivos que ayuden a los niños a aprender, jugar y entretenerse (TROSETH, RUSSO \& STROUSE, 2016) alimenta la producción e inunda el mercado. La oferta de contenidos interactivos, especialmente Apps para niños, ha crecido exponencialmente en los últimos años, y la industria digital crea recursos digitales para niños a un ritmo vertiginoso (solamente en la Apple Store existen hoy más de 80.000 Apps para el público infantil).

Pero que las Apps tengan una clasificación "infantil” incluso que tengan una etiqueta de "Apps educativas" impuesta por los mismos desarrolladores, tal y como subraya Zosh et. al (2016), no significa nada porque no se corresponde con la realidad. La etiqueta "educativa" debería implicar algún beneficio 
probado de estos recursos, pero, por el contrario, nadie realiza esta comprobación al incluir el calificativo en una App, ni los desarrolladores, ni los usuarios ni un comité independientes de expertos.

A menudo familias y educadores, para encontrar recursos educativos de calidad, recurren a bases de datos especializadas con evaluaciones ordenadas según temática y edad. Éstas se centran en los contenidos de los juegos y las aplicaciones, pero hoy sabemos que el diseño audiovisual e interactivo determina también la interacción del niño con las Apps (CRESCENZI \& GRANÉ, 2019).

Las clasificaciones de las Apps y juegos interactivos por edades, tiene un largo recorrido de estandarización incluso a nivel internacional, pero ¿son suficientes para prescribir una selección adecuada y de calidad?

Apps y juegos interactivos para la primera infancia, clasificaciones internacionales

La International Age Rating Coalition (IARC) implantó a nivel internacional un sistema de clasificación por edades para productos digitales, tales como videojuegos y aplicaciones móviles con la finalidad de "proteger" a los jóvenes, a partir de 2013. Este sistema es el que se emplea para clasificar todos los juegos y Apps para ordenadores, tablets y smartphones. Dependiendo del país de los desarrolladores la clasificación presenta variaciones, así en Europa se aplica la de Pan European Game Information (PEGI), que, por ejemplo, utiliza Google Play para la clasificación de Apps, a pesar de que en Estados Unidos es más común utilizar las clasificaciones Entertainment Software Rating Board (ESRB), usada por Windows Store; y además Apple Store ha creado su propio sistema Apple Rating. Más allá de estas clasificaciones globales, algunos países como Brasil aplican también el sistema de IARC mediante Classificação indicativa (CLASSIND).

\section{CLASIFICACIÓN}

PEGI

Pan European

Game Information
Presencia o ausencia de contenidos como: droga, desnudez, comportamientos o referencias sexuales, violencia física, lenguaje soez, miedo, discriminación, juego de azar, juego online.
Contiene

No contiene
ESRB

Entertainment

Software

Rating Board
Presencia o ausencia de contenidos como: violencia leve o irreal, violencia explícita, violencia intensa, lenguaje soez, lenguaje fuerte, blasfemias, temas indecentes, humor crudo, sangre mínima o abundante, juegos de azar, contenido sexual, droga.
No contiene

Infrecuente

Frecuente

Intenso 
Presencia o ausencia de contenidos como: episodios de violencia realista o no, violencia frecuente o intensa, blasfemia, contenido Apple Rating indecente, escenas de horror, contenido médico, uso de alcohol, tabaco o drogas, contenido sexual o nudismo, juegos de azar, acceso a webs externas no restringido.

No contiene

Infrecuente

Frecuente

Intenso

Fig. 01. Tabla resumen de las 3 clasificaciones internacionales.

Se observa como los tres sistemas clasifican la adecuación a cada edad a partir de la detección de contenidos negativos: lenguaje soez y/o blasfemias; discriminación; alcohol, tabaco y drogas (referencias o uso explícito); miedo; nudismo y sexo; violencia; conexión a internet sin restricción; juego de azar con apuestas. La clasificación PEGI se basa en la simple presencia o ausencia de estos contenidos (droga, desnudez, comportamientos o referencias sexuales, violencia física, lenguaje soez, miedo, ...), de forma que en caso de ausencia se estos contenidos, el juego se considera apto para todas las edades y se cataloga como PEGI 3. Es decir, el sistema basado en edades PEGI no tiene en cuenta realmente la edad porque no considera el nivel de dificultad ni las habilidades necesarias para jugar, no analiza la presencia de interferencias (publicidad, mensajes comerciales, etc. que se activan durante el juego), ni tampoco la presencia de mecanismos de protección infantil ante la conexión a Internet, o de protección de datos personal (fotos, vídeos, geolocalización, etc.).

Ninguno de los tres sistemas considera la adecuación de los objetivos ni los contenidos (aparte de la exclusión de los negativos), y tampoco ninguno considera la calidad y la adecuación de los sistemas interactivos y/o audiovisuales de las Apps.

Estos modelos internacionales para la clasificación de Apps no superan los límites de los anteriores sistemas de clasificación de videojuegos, sino que los reproducen. Consideran solamente un aspecto del contenido, la ausencia de contenido prejudicial, ignorando del todo la adecuación de los contenidos a los destinatarios potenciales y excluyendo el análisis de los sistemas interactivos y audiovisuales, y su acomodamiento a los jugadores. De manera que, aunque las clasificaciones se orientan a determinar una edad mínima para su uso, no garantizan que los destinatarios de la app puedan acceder o interactuar con ella, ni que comporte algún beneficio (aunque se defina como "educativa") o que no sea prejudicial por razones más sutiles que la presencia de desnudos o palabras soeces, como por ejemplo la perpetración de estereotipos de género o raza. No aportan ninguna información sobre la calidad de las aplicaciones y juegos, y establecen la edad de los destinatarios de forma arbitraria desde la propuesta de los desarrolladores.

Por ello resultan insuficientes para clasificar los contenidos por edades y no contribuyen con información sobre la calidad del contenido y del diseño interactivo de la aplicación. 
Apps para la primera infancia, estudios en evaluación de la calidad

Para que los recursos digitales sean herramientas potencialmente adecuadas para el juego y el aprendizaje de niños y niñas, es necesario que sean de calidad. La calidad de un contenido interactivo infantil está directamente relacionada con su adecuación a la edad, y específicamente al momento de desarrollo en que se encuentra el niño (GUERNSEY, 2012). Pero debido a la falta de información sobre las necesidades y las singularidades de los niños muy pequeños, los desarrolladores de juegos digitales a menudo no tienen en cuenta las características específicas de cada edad y momento del desarrollo infantil, lo cual tiene un impacto negativo en la calidad de sus productos. En este sentido, tal y como se ha planteado en anteriores estudios, el contenido de los materiales audiovisuales y multimedia no está separado de su forma, al contrario, el diseño interactivo de las aplicaciones para dispositivos móviles determina el acceso a los contenidos por parte de los usuarios (GRANÉ \& CRESCENZI, 2016).

Desde el grupo interuniversitario de investigación LMI (Learning, Media and social Interactions) se trabaja en una línea sobre infancia y pantallas, cuya finalidad es la búsqueda de la calidad de los recursos interactivos para la primera infancia con el objetivo de dar orientaciones a los desarrolladores como creadores y a los educadores como seleccionadores y prescriptores de los materiales.

La evaluación de la calidad de las Apps puede llevarse a cabo utilizando metodologías diversas, pero específicamente abarca dos paradigmas, el análisis heurístico de los materiales donde expertos en usabilidad definen cómo deben diseñarse las pantallas para niños y que aspectos deben considerarse en diseño interactivo; y las evaluaciones con usuarios que se llevan a cabo mediante test con usuarios potenciales, en este caso con niños menores de ocho años.

\section{Creación de un modelo teórico para el análisis de la calidad}

Bajo esta premisa se analizaron en profundidad los principios universales de diseño que intervienen directamente en entornos interactivos (GRANÉ, 2012) tales como Apps, videojuegos y entornos web. Una vez realizado el análisis se diseñó un estudio preliminar sobre los criterios de diseño interactivo aplicados directamente a las Apps infantiles y un análisis exploratorio observacional de los sistemas de evaluación y clasificación de las “mejores Apps educativas" para niños menores de 8 años, propuestos por educadores y expertos y presentados en 7 destacados portales y blogs internacionales, (GRANÉ \& CRESCENZI, 2016).

En una segunda fase se desarrolló un instrumento de observación de la calidad de las Apps infantiles con 108 ítems organizados en ocho dimensiones: Contenido y actividad, Diseño Visual (composición, distribución, atención, simplicidad visual), Interacción (usabilidad, ayudas, feedback, simplicidad), Sistemas de Navegación y Consistencia, Accesibilidad, Modelos Mentales y Referentes Culturales, Legibilidad, y Sonoridad. 
Este estudio inicial permitió corroborar la necesidad de integrar el diseño en la evaluación de la calidad de los recursos digitales infantiles, destacando en primer lugar la dimensión visual al considerar la composición equilibrada de las pantallas, el número de elementos y la disposición de los mismos para atender a la focalización del niño en el juego. En segundo lugar, un control adecuado de la complejidad visual de las pantallas, así como de la carga cognitiva de las acciones, y la adecuación a los modelos mentales y referentes culturales de los niños menores de 8 años. Y finalmente la relevancia de evitar interferencias en las Apps para la primera infancia (tales como mensajes o anuncios, así como elementos no necesarios y distractores).

A partir de este primer estudio preliminar se han llevado a cabo otras investigaciones relacionadas, en que se ha concretado el análisis de las Apps e interacciones niño-maquina desde el campo de la HCICCI (Human Computer Interaction - Children Computer Interaction).

\section{El análisis heurístico de las mejores Apps para la primera infancia}

Así, un segundo estudio consistió en el análisis de 100 Apps consideradas educativas para niños menores de 8 años seleccionadas mediante un muestreo teórico que incluyó las "mejores Apps educativas" presentadas por educadores y expertos en los siete destacados portales y blogs internacionales detectados en el estudio exploratorio (CRESCENZI \& GRANÉ, 2016). Estas bases de datos online suponen la principal fuente de información para familias y educadores para su elección de recursos infantiles digitales.

A partir de la ficha de observación diseñada en el estudio preliminar se siguió un procedimiento para la validación del instrumento que incluyó la participación de cuatro investigadoras y se midió la fiabilidad inter-codificadores utilizando el Kappa de Cohen y encontrando una concordancia alta para todas las variables. Posterior a la validación del instrumento se analizaron las 100 Apps para niños menores de 8 años, y los resultados del análisis pueden observarse desde tres perspectivas clave:

En primer lugar, bajo las dimensiones que corresponden al contenido, se mostró claramente el predominio de los contenidos curriculares, centrados en contenidos clave comunes en todo occidente, menoscabando otras dimensiones de aprendizaje relacionadas con los aspectos socioafectivos, artísticos, creativos y de construcción de conocimiento. Además, se observó la presencia de estereotipos y la falta de diversidad de los personajes en las Apps analizadas.

En segundo lugar, las dimensiones relativas al diseño visual mostraron problemas relacionados con la disposición y organización de elementos en pantalla que no permiten percibir un diseño global visualmente satisfactorio. En un 22\% de las Apps, no se consideraban los sistemas de realce para destacar elementos activos en el juego y también se observaron problemas de contraste figura - fondo en más de un 14\% de las Apps. Y a pesar de que la mayoría de las aplicaciones buscaban la simplicidad visual, en más del 78\% de las Apps no estaba claro el objetivo del juego, ni era intuitivo ni se indicaba claramente. 
Asimismo, uno de los problemas, que ya se detectaron en el primer estudio, fue la presencia de interferencias, como anuncios $(50 \%)$, textos innecesarios $(36 \%)$, y aparición de mensajes con enlaces externos $(9 \%)$.

Sobre las dimensiones referentes al diseño de la interacción se observaron dificultades relevantes en la atención a la simplicidad, debido a la elevada presencia de distractores y de elementos activos en pantalla, a menudo, innecesarios. Más de la mitad de la muestra incluía más de 4 elementos activos simultáneos, con una media de 18 y llegando a 39 en algún juego. Se analizaron también los sistemas de ayuda que, en un $25 \%$ eran innecesarios y en un $32 \%$ presentaban un formato incoherente con la edad de los destinatarios (por ejemplo, mensajes textuales). A pesar de que muchas de las Apps se dirigían a menores de 5 y a menores de 3 años, el texto era un elemento siempre presente, apareciendo incluso desde la pantalla inicial en el 96\% de los casos y siendo necesario para jugar en el 23\% de las Apps. Igualmente, incluso se utilizaba el texto como elemento del feedback en 23 de las 100 Apps analizadas. Y en el análisis de la adaptabilidad al target (menores de 8 años) se observó que solo el 22\% de las Apps educativas presentaban diferentes opciones de juego, con posibilidades de gestos diversos. Finalmente, en cuanto a la accesibilidad para la interacción de menores con diversidad funcional la ausencia de adaptaciones de cualquier tipo superaba el $90 \%$ de la muestra.

En resumen, los resultados mostraron que, desde las perspectivas de diseño visual e interactivo, de adaptabilidad, estructura y navegación, las "mejores 100 Apps" mostraban una escasa calidad y adaptación al desarrollo infantil (CRESCENZI \& GRANÉ, 2016).

\section{El análisis de las Apps con niños menores de cinco años}

El análisis heurístico de las aplicaciones mostró la necesidad de avanzar en la investigación de la calidad de los recursos desde la perspectiva, no de los expertos, sino de los usuarios. Es decir, de los niños.

Investigaciones anteriores (NACHER, ET. AL., 2014; ABDUL-AZIZ, 2013; HINIKER ET. AL., 2015) en el ámbito de la CCI han estudiado la adecuación de las tecnologías a los niños en edad preescolar analizando su comprensión del contenido interactivo, y observando que los menores de 6 , y especialmente los menores de 3 años, no consiguen comprender las instrucciones en las pantallas digitales, ni siquiera las instrucciones verbales; y que realizan menos acciones de las que son ofrecidas por las aplicaciones y videojuegos.

Este es el caso concreto de los gestos que hacen los niños pequeños en su interacción con pantallas. En el estudio de las 100 Apps "educativas", habíamos observado que los gestos que se proponían en ellas eran muchos: tap, doble tap, presionar y mantener, trazar una línea, scroll vertical, scroll horizontal, trazo circular, arrastrar y soltar, rascar, deslizar, rotar, usar más de un dedo, usar ambas manos, reducir y aumentar con dos dedos, pellizcar... Sin embargo, investigaciones anteriores, que habían experimentado con el gesto 
de los más pequeños ante las pantallas (ABDUL-AZIZ, 2013; VATAVU, CRAMARIUC \& SCHIPOR, 2014; CRESCENZI, PRICE \& JEWITT, 2014; CRISTIA \& SEIDL, 2015; NACHER, JAEN, NAVARRO, CATALÀ \& GONZALEZ, 2015), mostraban una variedad de gestos mucho menor llevados a cabo por los niños en sus interacciones digitales.

Bajo esta perspectiva llevamos a cabo una tercera investigación buscando aportar un conocimiento empírico sobre los procesos de interacción de los niños menores de 3 años. El objetivo se centró en explorar la interacción de los niños en edad preescolar con Apps de creación visual, estudiando la interacción espontánea y el gesto que realiza el niño delante la pantalla sin indicaciones ni orientaciones, sin interferencias, y sin límites de tiempo; todo ello en un entorno familiar escolar y con elementos de observación no invasiva.

La metodología de la investigación se basó en un diseño mixto secuencial, enmarcado en un paradigma pragmático, compuesto por dos estudios:

Un primer estudio de análisis del diseño interactivo de 32 Apps dirigidas a menores de 3 años para dibujar y colorear, usando el instrumento validado en la investigación anterior. Y un segundo mediante la observación de 21 niños de entre 14 y 33 meses mientras interactúan con Apps para tablet. Para ello, se escogieron dos actividades que los niños conocen y realizan normalmente sobre papel usando pintura de dedos: por un lado, dibujar, estudiando el gesto espontaneo y libre sobre la pantalla en blanco; y, por otro lado, colorear una figura para observar las posibles diferencias en el gesto y en el uso de herramientas.

Los resultados de esta investigación (CRESCENZI \& GRANÉ, 2019) mostraron que las aplicaciones de dibujo y pintura analizadas presentaban diversos problemas de diseño interactivo de la misma forma que se había indicado en anteriores análisis de Apps con diversos contenidos y actividades; además situaban las herramientas en espacios de la pantalla que no permiten el gesto factible de los participantes; y finalmente proponían un gran número de herramientas y opciones que se alejan completamente de las acciones y gestos que llevan a cabo realmente los menores de 3 años en su interacción espontanea con el medio.

Más reveladores fueron los resultados de las observaciones de los niños ante las pantallas. Los participantes iniciaban las interacciones mayoritariamente en la zona central de la pantalla, pero adaptaban su gesto al contenido de la app (dibujar sobre pantalla vacía o colorear una figura grande determina la zona que utilizan y el gesto que realizan). No se encontró una predominancia del gesto tap y se observó incluso una menor variedad de gestos durante la interacción espontanea, que en las investigaciones anteriores llevadas a cabo por otros investigadores estudiados. Así, los 21 participantes sólo utilizaron tres tipos de gesto: stroke, tap y press.

Es interesante destacar que los gestos y las acciones observadas no fueron uniformes entre los participantes, sino que evolucionan con la edad. Y esta evolución del gesto es un aspecto esencial a tener 
en cuenta en el desarrollo de Apps y la necesidad de simplificar el diseño adaptándolo a la edad de los usuarios.

\section{El análisis de Apps para niños con necesidades educativas especiales, la atención a la diversidad}

Una vez llevados a cabo los estudios heurísticos y con usuarios, se plantearon dos retos.

En primer lugar, la necesidad de un mayor número de evidencias empíricas sobre la interacción espontanea de los niños pequeños ante las pantallas, persiguiendo la finalidad de mejorar los recursos digitales.

Y, en segundo lugar, la obligación de centrar la atención en los menores con diversidad funcional o que tienen necesidades educativas especiales. Ello es debido a que los problemas con el diseño interactivo de las Apps para niños se hacen más evidentes y fuertes cuando son usados por aquellos que tienen dificultades o necesitan acercarse a los materiales de otra forma.

La posibilidad de acercar el diseño interactivo para la infancia a un modelo de diseño universal permitiría a todos los niños y niñas aprender desde sus potencialidades, así que este campo refuerza de manera firme la finalidad de la investigación en el campo de la CCI.

Este paradigma de Diseño Universal de las tecnologías de la información y la comunicación plantea usar un diseño visual e interactivo de las Apps único para todos, es decir, accesible y adaptable a todos los niños, aquellos con desarrollo típico y aquellos con necesidades educativas especiales. El concepto no tiene una relación directa con la perspectiva de Diseño Universal para el Aprendizaje, y se refiere a alcanzar unos niveles de accesibilidad óptimos para que un juego pueda ser usado por todos los niños y niñas.

Es un término sinónimo al concepto de accesibilidad o diseño sin barreras o diseño inclusivo (HASSAN y MARTIN, 2004). La idea inicial de la accesibilidad plantea que los diseños deben ser utilizables por diferentes personas con diferentes capacidades sin tener que ser adaptados. En definitiva, que cualquier persona pueda percibir, ver, entender, navegar, interactuar... con un material interactivo. Es una de las ideas clave en cualquier diseño digital, y toma más relevancia cuando hablamos del diseño de recursos para niños, porque la accesibilidad siempre se ha tratado como una atención a las personas con diversidad funcional, pero cada vez más es evidente que muchas adaptaciones pueden ser diseños para todos.

Por esta razón se llevó a cabo un cuarto estudio, cuyos resultados están actualmente en proceso de publicación. En él se utilizó un análisis preliminar para conocer todas las Apps orientadas a niños y niñas con necesidades educativas especiales disponibles mediante una búsqueda online sistematizada en cuatro idiomas (castellano, inglés, francés e italiano) hasta llegar a encontrar clasificaciones y evaluaciones independientes e internacionales realizadas por expertos. Posteriormente se seleccionaron 55 Apps dirigidas 
explícitamente al público infantil con habilidades funcionales diversas, mediante un muestreo no probabilístico por cuotas, de entre todas las consideradas muy buenas por los expertos.

Se realizó el análisis del contenido empleando la ficha de observación estructurada descrita en una anterior investigación (GRANÉ \& CRESCENZI, 2016) adaptándola a los objetivos de la investigación. Los resultados descubrieron una muestra muy heterogénea y sólo en algunos casos los productos se dirigían a mejorar una necesidad educativa concreta o apoyar una dificultad específica. No se pudieron detectar Apps con un diseño óptimo en todas las dimensiones estudiadas sobre contenido y actividades, diseño visual y diseño interactivo. Por el contrario, se hallaron necesidades de adecuación a la edad y al desarrollo de los destinatarios; además de insuficiencias en posibilidades de juego en grupo; asimismo se observó el continuado predominio del texto como sistema de comunicación en pantallas con grandes, y desaprovechadas, posibilidades audiovisuales; y finalmente se distinguió el escaso desarrollo de las opciones accesibles que permitirían ajustes en cada caso para usuarios diversos.

\section{Conclusiones y prospectiva}

Estos estudios coinciden en concluir que las aplicaciones para niños no siempre tienen en cuenta la edad, las singularidades cognitivas y el desarrollo específico del target al que se dirigen, y muchos de los diseños no solo no permiten la interacción, sino que incluso la dificultan. En este sentido cabe destacar que las tecnologías no pueden ser un problema para los niños sino una oportunidad para jugar, crear y aprender desde sus fortalezas y desde sus habilidades, más allá de sus dificultades.

Las investigaciones llevadas a cabo hasta el momento evidencian también que no existen criterios definidos para el diseño y la creación de Apps infantiles, de esta manera es claro que el recorrido todavía es muy largo para intentar llegar a un diseño educativo e interactivo de calidad. En este momento pueden realizarse, entre otras, algunas consideraciones que son necesarias:

- Promover la calidad del diseño visual, específicamente asegurar la simplicidad visual, la adecuada composición de pantalla y el diseño de la atención del usuario hacia los elementos de la misma.

- Trabajar el cuidado estético de todas las pantallas y elementos de las mismas.

- Planear el diseño de pantalla atendiendo al tamaño y número de elementos necesarios en cada actividad en adecuación al desarrollo cognitivo y motor de los usuarios finales.

- Cuidar los elementos musicales, sonoros y de voz en off de la misma forma que se cuida el diseño visual. Y programar alternativas visuales y de vibración a los mensajes sonoros, pero también a la inversa. 
- Evitar interferencias externas (anuncios o mensajes) e interferencias internas (elementos innecesarios en pantalla o distractores), así como la necesidad de reconfiguración durante el juego.

- Utilizar elementos de motivación extrínseca (gamification, recompensas, logros, ...) en las aplicaciones orientadas al aprendizaje.

- Fomentar la autonomía del niño en el juego y uso de la aplicación, mediante diseños intuitivos y naturales que no requieran de ayudas, o mediante sistemas de ayuda accesibles que respondan a diferentes formatos audiovisuales.

- Considerar diferentes gestualidades y uso de los dispositivos (tap, press, pinch, flick, ...) y posibilitar su adaptación según la edad de los destinatarios y la diversidad funcional.

- Permitir la adaptación de la vista del dispositivo (portrait or landscape).

- Ampliar las posibilidades de ajustes y adaptabilidad de los sistemas en: velocidades, dificultades y niveles; pero también en el formato (verbal, textual, sonoro, visual, i audiovisual) de los mensajes, instrucciones, y feedbacks de acierto y error que recibe el niño.

- Cuidar el tratamiento de las emociones a lo largo de toda la aplicación, desde las instrucciones, los elementos y personajes, la narración y los feedbacks.

- Crear sistemas de navegación adecuados a cada edad y a diferentes habilidades y capacidades.

- Considerar los modelos mentales y los referentes culturales de los niños en el planteamiento de temáticas, historias y personajes; pero también en el uso de iconos en las pantallas de juego.

- Trabajar desde la diversidad incluyendo personajes y elementos diversos, que se puedan personalizar, asegurando la representación de razas, genero, etc.

Pero a pesar de estas consideraciones se debe poner de manifiesto la necesidad de un modelo fiable y eficaz de análisis y selección de aplicaciones para niños, que asegure una adecuación del contenido y el diseño formal atendiendo a los modelos mentales y los procesos perceptivos de los destinatarios. Y ello será posible a medida que exista un avance en el estudio de la interacción de los más pequeños con las pantallas. Estudiar no solamente el gesto sino además las emociones, la comprensión, la consecución de los objetivos durante el juego, el tiempo de juego, la atención y focalización durante la acción, e incluso la verbalización y las interacciones con iguales, permitirá seguir avanzando para conseguir que existan recursos de calidad para la infancia. 


\section{Referencias}

ABDUL-AZIZ, N.A.B. Children's Interaction with Tablet Applications: Gestures and Interface Design. International Journal of Computer and Information Technology, v. 2, n. 3, 2013.

CLEMENTS, D.H. \& SAMARA, J. Young Children and Technology: What Does the Research Say? YC Young Children, v. 58, n. 6, p. 34-40, 2003.

CRESCENZI, L., JEWITT, C., \& PRICE S. The role of touch in preschool children's learning using iPad versus paper interaction. The Australian Journal of Language and Literacy, v. 37, n. 2, p.86-95, 2014.

CRESCENZI, L. \& GRANÉ, M. Análisis del diseño interactivo de las mejores Apps educativas para niños de cero a ocho años. / An Analysis of the Interaction Design of the Best Educational Apps for Children Aged Zero to Eight. Comunicar, v.46, p. 77-85, 2016. Disponible en: http://dx.doi.org/10.3916/C46-2016-08

CRESCENZI, L. \& GRANÉ, M. Touch Gesture Performed by Children under 3 Years Old When Drawing and Coloring on a Tablet. International Journal of Human-Computer Studies, v. 124, 2019. Disponible en: https://doi.org/10.1016/j.ijhcs.2018.11.008

CRISTIA, A. \& SEIDL, A. Parental Reports on Touch Screen Use in Early Childhood. PLoS ONE, v. 10, n.6, 2015. Disponible en: https://doi.org/10.1371/journal.pone.0128338

EU KIDS ONLINE. EU KidsOnline: findings, methods, recommendations. LSE, London, UK, 2014. Disponible en: http://eprints.lse.ac.uk/60512/

FIDLER, R. Tablets Are Now Commonplace in Households with Children. 2014 RJI Mobile Media Research Report. Donald W. Reynolds Journalism Institute (RJI), Mobile Media Poll, 2014. Disponible en: http://goo.gl/cIdYHn

FLEWIT, R., MESSER, D., \& KUCIRKOVA, N. New directions for early literacy in a digital age: The iPad. Journal of Early Childhood Literacy, v. 0, n. 0 1-22, 2014. Disponible en: https://doi.org/10.1177\%2F1468798414533560

GRANÉ, M. \& CRESCENZI, L. Modelo teórico para el diseño y evaluación de la calidad en las Apps infantiles (08 años). Digital Education Review (DER), 29, 2016. Disponible en: http://revistes.ub.edu/index.php/der/issue/view/1360

GRANÉ, M. El disseny interactiu a la xarxa. Barcelona: Publicacions de la Universitat de Barcelona, 2012.

GUERNSEY, L. Screen Time: How Electronic Media - From Baby Videos to Educational Software - Affects Your Young Child. NY: Basic Books, 2013.

HASSAN, Y. \& MARTÍN, F.J. Propuesta de adaptación de la metodología de diseño centrado en el usuario para el desarrollo de sitios web accesibles. Revista Española de Documentación Científica, v. 27, n.3, p. 330-334, 2004.

HINIKER, A., SOBEL, K.; HONG, S.R., SUH, H.; IRISH, I., KIM, D. \& KIENTZ, J.A. Touchscreen Prompts for Preschoolers: Designing Developmentally Appropriate Techniques for Teaching Young Children to Perform Gestures. IDC 2015 Medford, MA, USA, 2015.

KIRKORIAN, H.L., \& PEMPEK, T.A. Toddlers and touch screens: Potential for early learning? Zero to Three, v. 33, n. 4, p. 32-37, 2013.

LAURICELLA, A. R., PEMPEK, T. A., BARR, R., \& CALVERT, S. Contingent computer interactions for young children's objects retrieval success. Journal of Applied Developmental Psychology, v. 31, n.5, p. 362-369, 2010.

LANDIS, J., \& KOCH, G. The Measurement of Observer Agreement for Categorial Data. Biometrics, v.33, p. 159$174,1977$. 
MASCheroni, G., \& CUMAN, A., Net Children Go Mobile: Final Report (with country fact sheets). Deliverables D6.4 and D5.2. Milano: Educatt, 2014.

NACHER, V., JAEN, J., \& CATALA, A., Exploring visual cues for intuitive communicability of touch gestures to pre-kindergarten children. In: Conference: ACM International Conference on Interactive Tabletops and Surfaces. At Dresden. 2014.

NACHER, V., JAEN, J., NAVARRO, E., CATALA, A., \& GONZÁLEZ, P., Multi-touch gestures for prekindergarten children. International Journal of Human Computer Studies, v. 73, p. 37-51, 2015.

NIELSEN GROUP. Mobile Kids: the parent, the child and the smartphone. Nielsen's fourth-quarter 2017 Mobile Kids Report. 2017. Disponible en: https://www.nielsen.com/us/en/insights/article/2017/mobile-kids-the-parent-thechild-and-the-smartphone/

NÚCLEO DE INFORMAÇÃO E COORDENAÇÃO DO PONTO BR. TIC Kids online Brasil, Pesquisa Sobre o Uso da Internet por Crianças e Adolescentes no Brasil. 2017. Disponible en: http://globalkidsonline.net/wpcontent/uploads/2018/11/tic_kids_online_2017_livro_eletronico.pdf

READ, JC. \& MARKOPOULOS, P. Child-computer Interaction. International Journal of Child Computer Interaction, v.1, p. 2-6, 2013.

TROSETH, G., RUSSO, C., STROUSE, G. What's next for research on young children's interactive media? Journal of Children and Media, v. 10, n. 1, p. 54-62, 2016. Disponible en: https://doi.org/10.1080/17482798.2015.1123166

VATAVU, R. D., CRAMARIUC, G., \& SCHIPOR, D. M. Touch interaction for children aged 3 to 6 years: Experimental findings and relationship to motor skills. International Journal of Human-Computer Studies, v.74, p. 54-76, 2015.

VERNADAKIS, N., AVGERINOS, A., TSITSKARI, E., \& ZACHOPOULOU, E. The use of computerassisted instruction in preschool education: Making teaching meaningful. Early Childhood Education Journal, v. 33, n. 2, p. 99-104, 2005.

VINCENT, J. Mobile opportunities: Exploring positive mobile opportunities for European children. POLIS, LSE, London, UK, 2015.

ZOSH, J.M., HIRSH-PASEK, K., GOLINKOFF, R. M., \& PARISH-MORRIS, J. Learning in the Digital Age: Putting Education Back in Educational Apps for Young Children. Technology In Early Childhood Education. p. $1-5,2016$. 